\title{
Nanoparticle-modified metal-oxide-silicon structure enhancing silicon band-edge electroluminescence to near-lasing action
}

\author{
Ching-Fuh Lin, Peng-Fei Chung, and Miin-Jang Chen \\ Graduate Institute of Electro-Optical Engineering and Department of Electrical Engineering, National Taiwan University, \\ Taipei 106, Taiwan \\ Wei-Fang Su \\ Institute of Materials Science and Engineering, National Taiwan University, Taipei 106, Taiwan
}

Received September 10, 2001

\begin{abstract}
With the insertion of $\mathrm{SiO}_{2}$ nanoparticles in the oxide layer, near-lasing actions such as threshold behavior and resonance modes are observed at the Si bandgap energy of metal-oxide-silicon (MOS) structure. The threshold current is $\sim 12 \mathrm{~mA}$. The $\mathrm{SiO}_{2}$ nanoparticles cause simultaneous localization of electrons and holes to enhance phonon-assisted radiative recombination. Electroluminescence at Si bandgap energy is increased to orders of magnitude larger than in similar MOS structures without $\mathrm{SiO}_{2}$ nanoparticles. The efficient light emission at the Si bandgap energy indicates that a direct bandgap nature is not necessarily the basic requirement for radiative recombination. (C) 2002 Optical Society of America

OCIS codes: $230.3670,140.5960,140.2020,160.3380,230.0250$.
\end{abstract}

Because of the mature ultralarge-scale-integration technology based on $\mathrm{Si}$, Si has been the most important material for the integrated-circuit industry and is also attractive for applications in the emerging area of optoelectronics. Unfortunately, the indirect-bandgap nature of Si makes it inefficient as a light source, so optoelectronic integration on the Si chip is a challenging issue. Many efforts have thus been devoted to overcoming the indirect-bandgap obstacle. Those efforts include use of porous-silicon-based devices, ${ }^{1,2}$ doping impurities into $\mathrm{Si}^{3-5}$ use of $\mathrm{Si}^{+}$-implanted $\mathrm{SiO}_{2},{ }^{6}$ alloying $\mathrm{Si}$ with $\mathrm{Ge}$ or $\mathrm{Sn},{ }^{7,8}$ use of nanocrystalline Si (Refs. 9-11) or quantum-confinement structures ${ }^{12}$ deposition of polymer upon Si substrates, ${ }^{13}$ and growth of GaN upon Si substrates. ${ }^{14}$ Either the Si crystal is significantly modified or materials other than $\mathrm{Si}$ are used for light emission, so the emissions are not related to the Si bandgap energy. Recently, efficient light emission at the Si bandgap energy was achieved by introduction of dislocation loops or texturing silicon. ${ }^{15,16}$ Here we report that, by use of $\mathrm{SiO}_{2}$ nanoparticles in the oxide layer in metal-oxide-semiconductor (MOS) structures, the differential quantum efficiency of electroluminescence (EL) at the Si bandgap energy could be enhanced to nearly $10^{-4}$, orders of magnitude larger than that of similar MOS structures without $\mathrm{SiO}_{2}$ nanoparticles. ${ }^{17,18}$ Moreover, near-lasing actions at the Si bandgap energy, such as threshold behavior and resonance modes, are observed at room temperature.

The main hindrance to light emission from indirect-bandgap semiconductors is the momentum mismatch between electrons and holes, which leads to the low radiative recombination rate of electrons and holes. Several mechanisms for overcoming the momentum mismatch between electrons and holes in indirect-bandgap materials have been proposed. ${ }^{17}$ One of them is the spatial localization of carriers, which could have two effects: One is to cause the spread of carrier momentum as a result of the uncertainty principle and thus to relax the momentum mismatch between electrons and holes. The other is to cause the easy formation of excitons such that phonons can more easily be involved in the electron-hole radiative recombination. Either mechanism will change the optical matrix element to enhance the radiative recombination in indirect-bandgap materials. Lifetime measurement of electroluminescent MOS tunneling diodes has shown that the ratio for Shockley-Read-Hall, radiative, and Auger recombinations can be 1:0.196:0.096. ${ }^{19}$ Therefore the difference between the radiative recombination rate and the nonradiative recombination rate for indirect-bandgap materials can be less than 1 order of magnitude.

According to the theoretical model ${ }^{20}$ that explains the spectrum of electroluminescence from a MOS tunneling diode, phonons and excitons are both involved in radiative recombination. As an electron and a hole form an exciton, the probability of radiative recombination is increased. The reason for this is that the process is now more like a two-particle collision (phonon and electron-hole pair) than a three-particle (electron, hole, and phonon) collision. However, even if an electron and a hole do not form an exciton but are spatially confined together, they still have an increased probability of radiative recombination.

To spatially confine electrons and holes together, we used $\mathrm{SiO}_{2}$ nanoparticles in the insulation layer of the MOS structure. The processing steps were as follows: First the native oxide upon the $\mathrm{Si}$ wafer was removed. Then the solution with $\mathrm{SiO}_{2}$ nanoparticles was spun onto the wafer and dried in an oven. Figure 1 shows a field-emission type of scanning-electron microscope photo of $\mathrm{SiO}_{2}$ nanoparticles spun upon a $\mathrm{Si}$ wafer. Nanoparticles with a feature size of $\sim 12 \mathrm{~nm}$ are shown. After the nanoparticles were deposited, a 


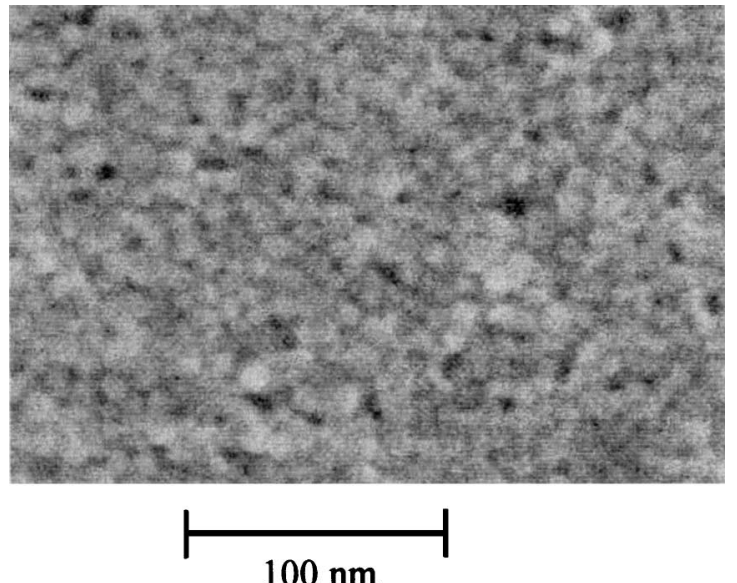

Fig. 1. Scanning-electron microscope photos of $\mathrm{SiO}_{2}$ nanoparticles spun upon a Si surface.

thin layer of $\mathrm{Al}$ metal was deposited upon the nanoparticles by evaporation. Then Ag paint was applied on top of the Al to hold a Au wire for electrical contact. A thick layer of $\mathrm{Al}$ was also evaporated onto the back side of the Si to form another electrical contact.

A schematic of the device is shown in Fig. 2. Because the spun $\mathrm{SiO}_{2}$ nanoparticles are not tightly packed, there are many hollow spaces between those particles. During evaporation, the $\mathrm{Al}$ atoms can get into those hollow spaces, so $\mathrm{Al}$ metal comes very close to the Si surface, which makes the structure similar to a metal-insulator-silicon structure. The measured I-V characteristics show that the device behaves similarly to a MOS tunneling diode. The measured EL spectrum is similar to previously reported spectra from MOS upon Si without nanoparticles, ${ }^{20,21}$ indicating that similar physics is involved in the radiative recombination. However, the measured efficiencies at $\mathrm{Si}$ bandgap energy are much larger than those previously reported for MOS structures without nanoparticles. ${ }^{17,18,21}$ More than 100 devices were measured. The differential quantum efficiency of measured EL from the periphery of the $\mathrm{Ag}$ paint pad on the devices varied from $1 \times 10^{-6}$ to $1 \times 10^{-4}$ at an injection current of $50 \mathrm{~mA}$. This efficiency does not take into account light blocked by the thick Ag paint. If the blocked light is also included, the best efficiency should be far beyond $10^{-4}$.

The temporal response of EL was measured for several samples as a square-current pulse was injected with a scanning-gate integrator-boxcar averager system. ${ }^{19}$ The measurements show that the rise time varies from 4.5 to $20 \mu \mathrm{s}$ and the fall time varies from 1.5 to $15 \mu \mathrm{s}$. Also, the longer rise time corresponds to a longer fall time and stronger EL emission. The nonradiative recombination states usually dominate the transition of electrons from the conduction band to the valence band and thus strongly influence the temporal response. More nonradiative recombination states speed up the recombination process and thus the temporal response. In the meantime, they also reduce the radiative emission. The varying rise and fall times indicate that those samples experience different amounts of nonradiative recombination states and so have different EL efficiencies. For $20 \mu$ s of rise time the nonradiative recombination rate is no more than $5 \times 10^{4} / \mathrm{s}$, implying that some portion of the Si wafer has good quality and could possibly serve for efficient radiative recombination.

With the enhanced radiative recombination rate, threshold behavior and resonance modes were observed. The light-current curve [curve (a) of Fig. 3] shows a sudden increase in output power after the threshold current $(\sim 12 \mathrm{~mA})$. The corresponding spectrum at $50 \mathrm{~mA}$ is shown in Fig. 4(a). Some of the resonance modes are clearly shown in Fig. 4(a). Both measurements were made for $\mathrm{cw}$ operation at room temperature. For comparison, the light-current curve of another device without threshold is shown by curve (b) in Fig. 3. Its output power has only a gradual increase with the injection current. Its corresponding spectrum is shown in Fig. 4(b). No resonance modes were observed for the devices without threshold behavior.

The output power shown in Fig. 3 was measured with the detector directly facing the device. Therefore the threshold shown in curve (a) of Fig. 3 is the behavior of the total output power, not only of the power of the sharp curves shown in Fig. 4(a). Because our monochromator (CVI, CM110) does not have sufficient resolution, only some resonance modes can be seen clearly. As a result, the spectrum of Fig. 4(a) appears to be a superposition of sharp spikes with a

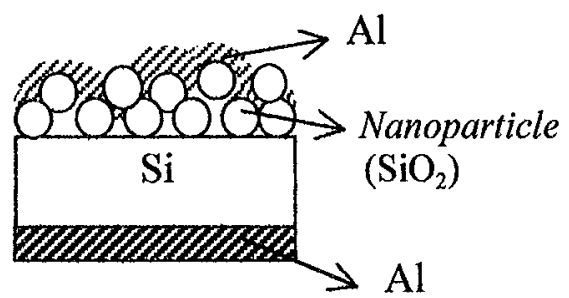

Fig. 2. Schematic of the $\mathrm{SiO}_{2}$ nanoparticle-modified MOS structure.

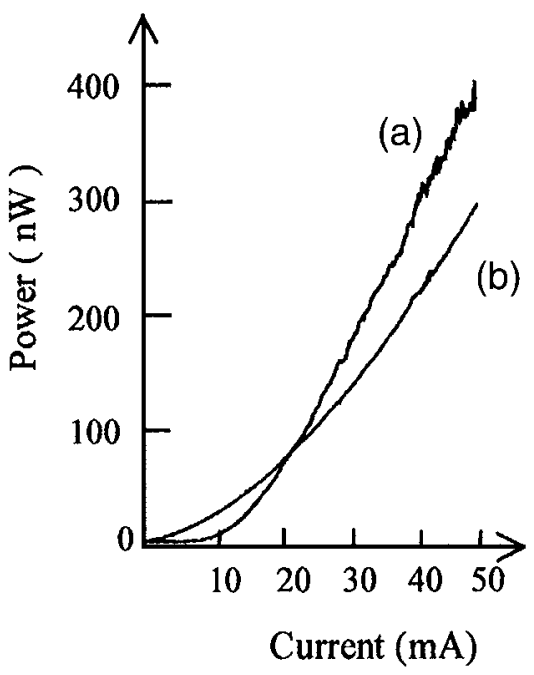

Fig. 3. Room-temperature light-current curves: (a) the device with threshold, (b) the device without threshold. 


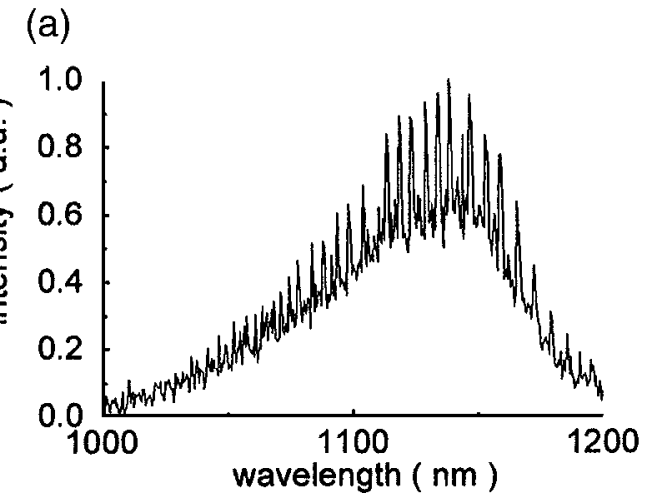

(b)

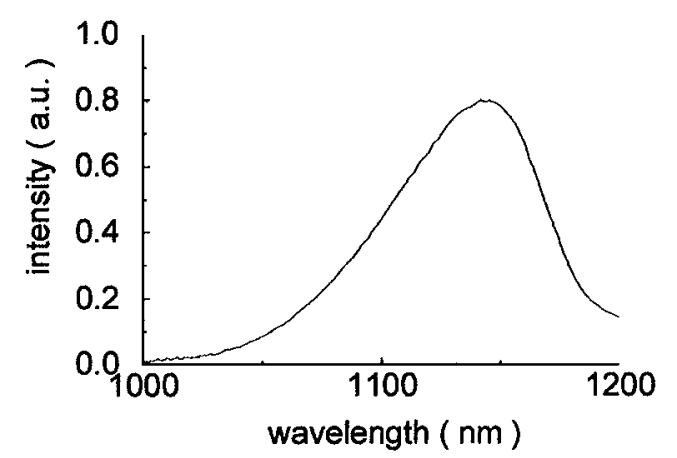

Fig. 4. Measured spectra: (a) with resonance modes, (b) without resonance modes. The spectra correspond to the Si bandgap energy.

broad background. The background in fact consists of many resonance modes that are not resolved by the monochromator.

The $\mathrm{SiO}_{2}$ nanoparticles cause the thickness of the insulation layer between the metal and Si to be nonuniform, as shown schematically in Fig. 2. The tunneling probability is greater, and band bending of $\mathrm{Si}$ is more severe, for the region with a thin insulation layer than in the thick region, so electrons and holes are spatially confined together near the $\mathrm{Si}-\mathrm{SiO}_{2}$ interface. Therefore radiative recombination is enhanced, as explained above. Also, the presence of $\mathrm{SiO}_{2}$ nanoparticles could lead to a local increase of the current density and so enhance radiative recombination. However, the local current density cannot be increased for orders of magnitude and so should not be the main reason for the enhanced EL.

Because $\mathrm{SiO}_{2}$ nanoparticles induce no damage to the $\mathrm{Si}$ crystal, nonradiative centers remain low for the portion of a Si wafer that has good quality. The near-lasing actions occur probably because the optimal enhancement of radiative recombination by the $\mathrm{SiO}_{2}$ nanoparticles and the minimal nonradiative recombination states coincidentally occur at the same spot on the Si wafer, resulting in significant gain. Also, the high reflection of the top Ag-paint pad and the bottom Al layer provides the optical feedback for resonance. In addition, the small absorption of $\mathrm{Si}$ at the bandgap energy causes the gain possibly to surpass the absorption loss of the substrate, leading to the near-lasing actions.
In conclusion, EL at the Si bandgap energy from a MOS structure is greatly enhanced by use of $\mathrm{SiO}_{2}$ nanoparticles for the oxide layer. The reason is that the $\mathrm{SiO}_{2}$ nanoparticles cause electrons and holes to be localized, making the radiative recombination of an electron-hole pair more like a two-particle collision than a three-particle collision. The measured differential quantum efficiency of EL could be near $10^{-4}$, even with significant light blocked by the $\mathrm{Ag}$ paint. In addition, for the first time to our knowledge, nearlasing actions such as threshold behavior and resonance modes that correspond to Si bandgap energy have been observed.

This research is supported in part by National Science Council, Taipei, Taiwan, under contract NSC90-2622-L-002-002. C.-F. Lin's e-mail address is cflin@cc.ee.ntu.edu.tw.

\section{References}

1. A. G. Cullis and L. T. Canham, Nature 353, 335 (1991).

2. M. V. Wolkin, J. Jorne, P. M. Fauchet, G. Allan, and C. Delerue, Phys. Rev. Lett. 82, 197 (1999).

3. G. Franzo, F. Priolo, S. Coffa, A. Polman, and A. Carnera, Appl. Phys. Lett. 64, 2235 (1994).

4. M. Matsuoka and S. Tohno, Appl. Phys. Lett. 71, 96 (1997).

5. S. G. Pavlov, R. Kh. Zhukavin, E. E. Orlova, N. V. Shastin, A. V. Kirsanov, H.-W. Hubers, K. Auen, and H. Riemann, Phys. Rev. Lett. 84, 5220 (2000).

6. W. Skorupa, R. A. Yankov, I. E. Tyschenko, H. Frob, T. Bohme, and K. Leo, Appl. Phys. Lett. 68, 2410 (1996).

7. S. Oguz, W. Paul, T. F. Deutsch, B.-Y. Tsaur, and D. V. Murphy, Appl. Phys. Lett. 43, 848 (1983).

8. C. W. Liu, J. C. Sturm, Y. R. J. Lacroix, M. L. W. Thewalt, and D. D. Ferovic, Mater. Res. Soc. Symp. Proc. 342, 37 (1994).

9. D. B. Geohegan, A. A. Puretzky, G. Duscher, and S. J. Pennycook, Appl. Phys. Lett. 73, 438 (1998).

10. L. Pavesi, L. Dal Negro, C. Mazzoleni, G. Franz, and F. Priolo, Nature 408, 440 (2000).

11. M. H. Nayfeh, N. Barry, J. Therrien, O. Akcakir, E. Gratton, and G. Belomoin, Appl. Phys. Lett. 78, 1131 (2001).

12. F. Buda, J. Kohanoff, and M. Parrinello, Phys. Rev. Lett. 69, 1272 (1992).

13. D. R. Baigent, R. N. Marks, N. C. Greenham, R. H. Friend, S. C. Moratti, and A. B. Holmes, Appl. Phys. Lett. 65, 2636 (1994).

14. R. Birkhahn and A. J. Steckl, Appl. Phys. Lett. 73, 1700 (1998).

15. W. L. Ng, M. A. Lourenco, R. M. Gwilliam, S. Ledain, G. Shao, and K. P. Homewood, Nature 410, 192 (2001).

16. M. A. Green, J.-H. Zhao, A.-H. Wang, P. J. Reece, and M. Gal, Nature 412, 805 (2001).

17. C.-F. Lin, C. W. Liu, M.-J. Chen, M. H. Lee, and I. C. Lin, J. Appl. Phys. 87, 8793 (2000).

18. C. W. Liu, M. H. Lee, M.-J. Chen, I. C. Lin, and C.-F. Lin, Appl. Phys. Lett. 76, 1516 (2000).

19. M.-J. Chen, C.-F. Lin, M. H. Lee, S. T. Chang, and C. W. Liu, Appl. Phys. Lett. 79, 2264 (2001).

20. M.-J. Chen, E.-Z. Liang, S.-W. Chang, and C.-F. Lin, J. Appl. Phys. 90, 789 (2001).

21. M.-J. Chen, C.-F. Lin, W. T. Liu, S. T. Chang, and C. W. Liu, J. Appl. Phys. 89, 323 (2001). 